\title{
La formación de terapeutas ocupacionales desde un interés crítico de la Educación
}

The formation of occupational therapists from a critical interest of the Education

\author{
Irene Muñoz Espinosa*
}

\section{Resumen}

Este ensayo presenta un análisis acerca de la formación de terapeutas ocupacionales, fundamentada en el interés crítico y emancipador, que nos describe el filósofo alemán Jürgen Habermas, y el planteamiento del educador Paulo Freire. Formación que favorece aquellos contenidos y objetivos que desarrollan la autonomía, libertad y reflexividad de los estudiantes.

El propósito es facilitar el conocimiento de la realidad y la experiencia diaria, tanto personal como profesional, de modo de aportar desde la Terapia Ocupacional, a la tarea de la construcción de una sociedad más democrática, solidaria, justa, libre e igualitaria.

Palabras clave: formación terapeutas ocupacionales- interés critico y emancipador .

\begin{abstract}
This essay presents an analysis about the formation of occupational therapists, based on the criticize and emancipation interest, that german philosopher Jürgen Habermas describes to us, and also the exposition of the educator Paulo Freire. Formation that focus on those contents and objectives that develop the autonomy, freedom and reflection of the students.

The intention is to facilitate the knowledge of the reality and the daily experience, personal as well as professional, as a way to contribute from the Occupational Therapy to the task of the construction of one more democratic, shared in common, right, free and equal society.
\end{abstract}

Key words: formation - occupational therapists - criticize and emancipation interest.

\footnotetext{
* Terapeuta Ocupacional. Orientadora Familiar. Licenciada en Ciencia de la Ocupación. Magíster@ en Educación en Ciencias de la Salud. Académico. Profesor Asistente. Facultad de Medicina. Universidad de Chile. Dirección: Av. Independencia 12027. Santiago de Chile. Fono: 56-02-9786588. e-mail: imunoz@med.uchile.cl
} 


\section{CONTEXTO EN QUE SE DESARROLLA LA FORMACIÓN DE TERAPEUTAS OCUPACIONALES}

"La discapacidad y vulnerabilidad de distintos grupos sociales requiere de profesionales capaces de crear herramientas y estrategias para integrarlos a la comunidad" (1). Frente a este desafío los terapeutas ocupacionales, como profesionales del área de la salud, requieren de una formación académica que les permita desarrollar estrategias tendientes a favorecer la integración y participación social de estos grupos, a través del abordaje del contexto y del desempeño ocupacional de las personas.

La Terapia Ocupacional fundamenta sus intervenciones en la Ocupación ${ }^{1}$ que desempeñan los seres humanos en su cotidianeidad. El ser humano al involucrarse en ocupaciones, puede modificar su desempeño, participando de actividades significativas que, por consiguiente, afecten la salud, el bienestar y su satisfacción con la vida. (2)

Es en estos fundamentos, que el proyecto educativo de la Escuela de Terapia Ocupacional de la Universidad de Chile, desarrolla un proceso permanente de reflexión y construcción colectiva, con espacios de participación, que permitan el desarrollo humano de los docentes y estudiantes. Lo importante es favorecer una educación con calidad, que permita dar respuesta a las necesidades actuales de salud del país, por lo que es necesario rescatar la tendencia de superar el umbral que separa el paradigma positivista por un paradigma interpretativo o hermenéutico. Esto debe reflejarse en las nuevas estrategias de formación que requieren los futuros terapeutas ocupacionales, por lo que es necesario adoptar un enfoque que permita la identificación de las situaciones o fenómenos de la realidad social en los cuales se desempeña el ser humano y que se constituyen en su objeto de estudio

Para entender el sentido complejo de la realidad social humana y por ende, lo complejo de la Ocupación humana, es necesario adoptar más bien el punto de vista opuesto al postulado por la epistemología clásica. Es preciso, entender la complejísima trama, que surge más allá de lo objetivo y que hace necesario rescatar las subjetividades de los sentidos y significados ${ }^{2}$ de la ocupación. Se trata de un espacio en el que el aspecto objetivo de lo real, y su aspecto subjetivo, sólo están para efectos de aprendizaje, separando el cuerpo y alma de la persona. Existen diferentes formas de interpretar la ocupación de las personas, desde la epistemología hermenéutica, es posible conocerla a través de la interacción entre el observado y el observador; a diferencia de la clásica positivista en que estudiamos la ocupación como conductas, acciones predecibles y objetivas.

Cada ser humano es un ser único, que tiene su propia forma de interpretar la realidad, desde esta mirada se requiere comprender la Ocupación como un elemento central del proceso de intervención de Terapia Ocupacional, que facilita al individuo el involucrarse en actividades que apoyen su participación en la vida cotidiana

\footnotetext{
1 "Ocupación es aquella actividad con sentido en la que la persona participa cotidianamente y que puede ser nombrada por la cultura". Comité de Ciencia de la Ocupación. Escuela de Terapia Ocupacional. Universidad de Chile. 2007

${ }_{2}^{2}$ El sentido se refiere al "valor" personal o individual que se da a las actividades, el que se construye desde las experiencias sensoriales, afectivas, motoras, cognitivas, sociales, a lo largo de la vida. Comité de Ciencia de la Ocupación. Escuela de Terapia Ocupacional. Universidad de Chile. 2007.
} 
El involucrarse en ocupaciones ocurre en una variedad de contextos (culturales, físicos, sociales, personales, temporales, espirituales y virtuales) (2), por lo tanto se reconoce que la experiencia individual y el desempeño no pueden ser entendidos o adoptados sin la comprensión de la variedad de contextos en los que las ocupaciones y actividades de la vida diaria ocurren. Por lo que la formación de Terapeutas Ocupacionales requiere que los estudiantes estén atentos a las situaciones de exclusión que se dan de los grupos vulnerables de nuestra sociedad y por sobre todo, es necesario que logren crear condiciones que permitan favorecer la justicia ocupacional, la dignidad y la solidaridad por el otro. Por lo tanto estamos enfrente de un compromiso moral, al formar a los estudiantes que confían su formación en nuestra Escuela, un compromiso directamente con ellos y con la sociedad.

Para esto es necesario que el programa de formación se fundamente en el interés crítico y emancipador, que nos describe Habermas (3), que permita a los estudiantes comprender la Ocupación no como un objetivo, sino más bien como un fenómeno tan variable y diverso como es el propio ser humano. El interés emancipatorio, que produce el conocimiento crítico es fundamental en el estudio de la Terapia Ocupacional. A través de este interés se facilita la autorreflexión y se genera la crítica que permite a los estudiantes desarrollar un saber crítico sobre la Ocupación del ser humano, de modo que éstos vayan convirtiéndose en profesionales reflexivos y no en técnicos de terapia ocupacional

Como sabemos, la ciencia social crítica, según Habermas (4) busca hacer a los seres humanos más conscientes de sus propias realidades, más críticos de sus posibilidades y alternativas, más confiados en su potencial creador e innovador, más activos en la transformación de sus propias vidas, en una palabra, más autorrealizados como tales, para que sean ellos los forjadores de su propio destino. Es por esto que necesitamos introducir la capacidad reflexiva en los estudiantes, de modo de que puedan comprender e intervenir sobre la realidad de un modo crítico y sean a futuro terapeutas ocupacionales capaces de aportar a la transformación social.

\section{SIGNIFICADO DE LA FORMACIÓN DE TERAPEUTAS OCUPACIONALES}

La vulnerabilidad de los grupos minoritarios (personas en condición de pobreza, discapacidad, cesantía, alienación) de nuestro país, así como de Latinoamérica son cada vez mayores. El libre mercado y el mundo globalizado excluyen cada vez a más grupos de personas que no pueden ingresar a este ritmo, ya sea por un impedimento físico, mental y /o social. Es así, como desde estos últimos años la Terapia Ocupacional ha tenido que avanzar desde su mirada reduccionista de atender sólo la función versus la disfunción, hacia una perspectiva que amplia su horizonte hacia la concepción de integridad del ser humano, considerando su historicidad y contexto social.

De esta forma nos comenzamos a preguntar ¿con cuál mirada vamos a enfocar al ser humano en su integridad? Pues es necesario el cambio de paradigma, ya no sólo seremos observadores de la realidad, sino que tendremos que asumir nuestra participación e interpretación de ella. Asumimos nuestro rol de constructores de esta sociedad, de la cual muchas veces renegamos, sin embargo formamos parte de su compleja estructura y función. 
De acuerdo a lo anterior, el significado que tiene la orientación social -crítica para la formación de terapeutas ocupacionales, es importantísima, considerando su principio de favorecer la capacidad autorreflexiva del ser humano inserto en su mundo social , que permite que los estudiantes sean capaces de generar un cambio de paradigma, que les permita ver el estudio de la Ocupación, como una actividad compleja del ser humano, que no es objetiva y que requiere de que ellos desarrollen el interés por investigar aquellos fenómenos que los asombran y que son parte de su propia realidad.

De este modo es necesario apostar por un tipo de educación crítica, liberadora, que nos dé la posibilidad de desencadenarnos del pensamiento único y objetivo, para ser capaces de conocer, contrastar, mejorar y transformar las teorías personales que construimos en nuestra vida cotidiana, de modo que nos permitan entender la realidad y seamos capaces de actuar en la misma.

En la constante reflexión crítica de la formación de los estudiantes, es donde muchas veces nos encontramos con la dimensión ética de la práctica educativa la cual tiene dos dimensiones (5): primero que el acto de enseñar presupone "que algo de valor va a ser enseñado” y, segundo, las relaciones entre docentes y estudiantes son "inherentemente relaciones de desigualdad”. Estos dos cuestionamientos suponen asumir algún principio moral ético que regula a los actores (estudiantes y docentes). Es esta reflexión constante la que nos permite poder guiar nuestros pasos como docentes, de modo de poder generar un programa de formación que responda a las necesidades de los estudiantes como futuros terapeutas ocupacionales. Sabemos que tenemos el poder del académico, pero a la vez confiamos en que los valores que queremos transmitir son fundamentados de acuerdo a los principios humanistas de nuestra profesión.

Como lo señala Paulo Freire, es primordial considerar que tras cualquier práctica educativa siempre hay una visión del ser humano, del mundo y de la sociedad (6). Como docentes no podemos asumir una neutralidad en nuestro que hacer y es necesario cuestionar la realidad histórica, sociológica y cultural en las cual estamos inmersos como terapeutas ocupacionales y por sobre todo como educadores. Tenemos el sueño, al igual el que tuvo Freire, de transformar la realidad y construir entornos más justos, especialmente para los grupos más vulnerables de nuestra sociedad, considerando los principios éticos en nuestras prácticas docentes.

Es por esto que la formación de terapeutas ocupacionales, se basa en los principios del Humanismo, que nos plantea Carl Rogers "El individuo posee en sí mismo potenciales recursos para su propia comprensión, para cambiar su autoconcepto, sus actitudes, y para dirigir su conducta, y estos recursos pueden ser liberados a condición de que un determinado clima de actitudes psicológicas facilitadoras pueda ser logrado" ${ }^{3}$.

Para esto es necesario apostar a la Autenticidad, la Aceptación incondicional y la Empatía que nos plantea como actitudes necesarias a desarrollar, no sólo como terapeutas, sino como seres humanos. De esta forma aportamos a la humanización de la docencia y por ende, a humanizar la formación, esto es, que los estudiantes se motiven por la búsqueda de autonomía, desarrollen una mayor conciencia sobre el mundo y así generen la capacidad crítica y transformadora.

\footnotetext{
${ }^{3}$ Rogers, C. y Mariam Kinget (1971) Psicoterapia y relaciones humanas. Tomo I Madrid- Barcelona Ed. Alfaguara. Pág. 108
} 
El papel del docente en la formación de terapeutas ocupacionales, es de un facilitador de los procesos reflexivos de los estudiantes, que permita que éstos, sean los protagonistas activos de su proceso educativo; y a la vez el docente "es enseñado" con la retroalimentación y autorreflexión que surge en el diálogo del proceso.

Esta tarea no es sólo del estudiante, sino que es nuestro mayor desafío, no podemos predicar sin practicar, es por esto que tenemos que desarrollar nuestra propia reflexión crítica acerca de nuestras prácticas docentes.

\section{PROPÓSITOS E INTENCIONES}

El programa de formación de terapeutas ocupacionales está iniciando su proceso de reformulación hacia una formación focalizada en competencias. Las competencias que se esperan de los Terapeutas Ocupacionales, se basan en los principios del humanismo y del interés social crítico; en la cual se requiere que los estudiantes se vayan acercando a ser personas comprometidas socialmente, competentes, con una alta responsabilidad ética, con espíritu investigador y deseosos de desarrollar una educación permanente.

La formulación de competencias para el terapeuta ocupacional, nos exige realizar un análisis de la realidad social y del desempeño ocupacional de los seres humanos en ella. Visualizar las competencias necesarias, que se nos imponen para estar dentro y no afuera de nuestro medio social es una gran tarea; tenemos que comenzar por convertir la información en conocimiento útil, tener iniciativa, desarrollar la capacidad para abordar el reconocimiento, análisis y proyección de los problemas cotidianos desde una perspectiva multidimensional. Es necesario además, tener la capacidad de trabajar en equipos transdisciplinarios, reconociendo otros lenguajes y visiones de la realidad, construyendo un lenguaje común, ser capaces de establecer diálogos, que nos permitan poner en juego el pensamiento complejo que Morin (7) nos presenta como la capacidad de "ejercitarse en un pensamiento capaz de tratar, de dialogar, de negociar, con lo real".

\section{ESTRATEGIAS METODOLÓGICAS}

Las metodologías de enseñanza favorecen los aprendizajes reflexivos, que fomentan la autonomía y la reflexión de los estudiantes; favoreciendo que el conocimiento surja desde la experiencia de ellos, de sus necesidades y que orienten el diálogo hacia la construcción de un conocimiento compartido.

Las actividades articulan la teoría con la práctica, a través de módulos de autoinstrucción, tutorías guiadas en pequeño grupo con los docentes participantes de las asignaturas y el desarrollo de diarios reflexivos. Las Prácticas Integradas, que los estudiantes desarrollan desde primer año a lo largo de toda la carrera, les permiten en terreno interactuar con usuarios de diferentes instituciones comunitarias y hospitalarias. Es en esta instancia que los estudiantes integran, relacionan, analizan y asocian los conocimientos desarrollados en las distintas asignaturas correspondientes a su nivel. Esto con el fin de comprender el fenómeno de la ocupación humana desde lo teórico a lo práctico y viceversa. 
En las clases y tutorías intentamos generar el máximo de diálogo posible, basándonos en los que Paulo Freire (6) nos presenta: "el diálogo como método de construcción solidaria del conocimiento”, "el diálogo implica la relación entre sujetos que tienen experiencias que interactúan para aprehender más la naturaleza de un objeto que van analizar”.

La construcción de procesos de diálogos tiene que ver con el trabajo por la democratización de los mismos, donde se da una coparticipación de todas las personas relacionadas con esa acción educativa. Es por esto que intentamos trabajar en pequeño grupo a través de tutorías de modo de generar la participación de todos los estudiantes, en diálogo y retroalimentación constante con el grupo y el docente.

Pero para que eso se produzca hemos de tener en cuenta, como nos señala Freire, el diálogo como un encuentro sincero e igualitario entre docente y estudiante. Para que se de lugar a este encuentro es necesario que todos los involucrados en el proceso, se reconozcan en su saber y en su ignorancia. La apuesta es por una educación de la pregunta, que facilite la curiosidad del estudiante y favorezca los procesos de indagación que nos permitan conocer y crear nuevos conocimientos. Convertir los espacios educativos y formativos en lugares de reflexión, de estudio, de debate y de construcción del conocimiento, generando círculos auténticos de cultura constituidos por todos los agentes involucrados en el proyecto educativo y social, es la clave para aprendizajes transformadores, que permitan producir cambios en nuestra sociedad.

Como docentes, confiamos en que los estudiantes tienen la capacidad desarrollar una postura crítica, sin embargo muchas veces nos decepcionamos de ello, por la débil participación que generamos en los grupos. Quizás, ahora analizando más profundamente, la poca confianza que ellos nos tienen, surge desde nuestra posición vertical, al tener nosotros los docentes el poder de la evaluación, el que claramente muchas veces es el destructor del diálogo horizontal

Una vez más surge un gran tema para reflexionar, ¿cómo lograr la capacidad humana de ser una persona confiable para el estudiante?, tal vez la respuesta la encontremos en lo que Freire (8), nos describe como la capacidad de amar, " nuestra razón muchas veces nos distancia de nuestra emoción y esto nos aleja de lo humano de la educación, son el amor y los afectos los que nos permiten la interacción con el mundo”, Freire nos señala con certeza "es necesario amar para educar, aprender y enseñar". Este es el inicio de una gran reflexión en nuestra formación como docentes, ¿cuánto amamos la docencia, cómo podemos desde este amor crear instancias de diálogo y aprendizajes reflexivos con los estudiantes.

\section{EL PROCESO DE EVALUACIÓN}

Reflexionando en torno a la tan inquietante evaluación, desde una teoría centrada en la persona y en el interés social crítico; es necesario considerar cuáles son los requisitos que, desde esta postura, se deberían tener en cuenta para hacer de este proceso un resultado efectivo. Primero que nada tenemos que tener claro la congruencia que debiera existir entre la teoría y la práctica educativa; y cómo se plasman estos principios en lo cotidiano de las clases, tutorías y por sobre todo las evaluaciones que 
deben rendir los estudiantes, para ser considerados aprobados o reprobados de las asignaturas.

En esta instancia evaluativa, que tiene por objeto calificar los conocimientos, conductas, y actitudes del estudiante, debieran surgir las potencialidades y procesos de reflexión crítica que lo llevan a la toma de conciencia de sí mismo y de la realidad.

La evaluación es mucho más que una tecnología, una metodología y una medición. La evaluación debería abordar lo profundo del significado del actuar del estudiante, más que el logro de objetivos. Es necesario revisar los criterios que permiten desarrollar en forma eficiente este proceso y no caer en el tecnicismo que provoque las tan duras injusticias de la evaluación.

La metodología de trabajos escritos y exposiciones de temas en forma grupal, permiten generar diálogo y reflexión entre los estudiantes, sin embargo; existen debilidades en la forma de evaluar esos procesos, ya que los criterios se centran en la capacidad de generar discusión y reflexión, junto al análisis y comprensión del fenómeno de la Ocupación Humana. Si bien esto lo hemos logrado medir y transformar en un número, surge la inquietud de cómo evaluar la capacidad de los estudiantes de ser críticos y transformadores de la sociedad. Esta posibilidad surge en las prácticas integradas, momento en el cual el estudiante ponen en juego todas sus capacidades y procesos de reflexión, para generar programas de intervención de T.O, transformadores que respondan a las necesidades de igualdad y oportunidades de los usuarios con los cuales trabajan. Este momento es evaluado a través del diario reflexivo, técnica que permite que el estudiante lleve un registro de su proceso de práctica, en la cual se construyen diálogos entre la teoría con su práctica profesional. La construcción del diario reflexivo va apoyado por el diálogo establecido entre el grupo de estudiantes y el docente tutor, quienes facilitan la reconstrucción de la experiencia y el análisis y comprensión de ésta. A la vez en su sistematización de experiencia de práctica, que es un trabajo grupal, los estudiantes deben ser capaces de comprender, analizar y fundamentar su programa de T.O, desde un enfoque que se centre en un interés emancipador.

La evaluación del desempeño individual se realiza a través de una pauta de desempeño en la cual se miden el logro de diferentes aspectos (conocimientos, actitudes, procedimientos); sin duda que es una pauta que deberá analizarse y reflexionarse para que cumpla con ser coherente con la formación del estudiante.

Cabe destacar que en todas las formas de evaluación, está presente la evaluación formativa, en la cual a través del diálogo se reconstruyen los análisis de modo que los estudiantes puedan reconocer sus falencias y fortalecer aún más sus virtudes.

\section{Reflexiones}

El rol de los docentes formadores de terapeutas ocupacionales, requiere de constantes análisis reflexivos y críticos enfocados hacia la práctica como educadores. Como nos plantea Freire, "el espacio pedagógico es un texto para ser constantemente leído, interpretado, escrito y reescrito"4

4 Freire, Paulo. Pedagogía de la autonomía. Madrid: Siglo XXI 1997. pág. 94. 
Es necesario continuar profundizando en nuestra formación académica y en nuestras prácticas docentes, de modo de llegar a ser más eficaz en el desarrollo del diálogo, que nos permita propiciar espacios educativos que generen la reflexión entre los estudiantes y docentes; de modo de favorecer el entendimiento, y llegar a construir espacios de beneficio común.

A la vez es un desafío constante seguir validando el espacio universitario, como un ámbito ideal para el diálogo, es aquí donde se pueden favorecer las oportunidades de expresar, discutir, compartir y fortalecer o debilitar actitudes, virtudes y hábitos humanos como la solidaridad, comprensión, pluralismo, cooperación para el bien común y público. De modo de generar instancias realmente transformadoras de nuestra sociedad, en la cual los terapeutas ocupacionales aporten a la tarea de la construir en este mundo globalizado una sociedad más democrática, solidaria, justa, libre e igualitaria.

Este artículo reflexiona en torno a la necesidad de incorporar en la formación de los terapeutas ocupacionales, los fundamentos necesarios que permitan atender las necesidades actuales de nuestra sociedad y que dialoguen constantemente con los intereses críticos y emancipatorios de la educación. Tenemos que estar atentos a la comprensión de las transformaciones sociales, culturales, económicas, políticas y sanitarias de modo de problematizar constantemente los dominios de formación del terapeuta ocupacional de nuestra Escuela, que guiarán su práctica profesional y le permitirán el diálogo constante con la realidad.

Esperamos que los terapeutas ocupacionales de la Universidad de Chile logren la capacidad de comprender e intervenir sobre la realidad de un modo crítico, transformando la realidad favoreciendo el desarrollo de la profesión y de la investigación en Ciencia de la Ocupación.

\section{BIBLIOGRAFÍA}

(1) Universidad de Chile. Facultad de Medicina. Escuela de Terapia Ocupacional. <http://www.med.uchile.cl/escuelas/terapia/historia.html> [consulta :15 abril 2007]

(2) Asociación Americana de Terapia Ocupacional. "Marco de Trabajo para la Práctica de la Terapia Ocupacional: dominio y proceso". Revista Americana de Terapia Ocupacional (AJOT) 56, (2002) pág. 609-639.

(3) Habermas, J. Teoría de la Acción Comunicativa, vol. I: Racionalidad de la acción y racionalización social. Madrid, Taurus, 1987.

(4) Habermas, J. Teoría de la Acción Comunicativa, vol. II: Crítica a la razón funcionalista. Madrid, Taurus, 1987.

(5) Angulo, F. Blanco, N. (coords) 1994 Teoría y desarrollo del curriculum. Málaga: Aljibe. Cap.6 págs 117-118. 
(6) Freire, Paulo. La Naturaleza política de la educación. Cultura, poder y liberación. Madrid: Piados 1990. pág. 118 -119

(7) Morin, Edgar. Introducción al pensamiento complejo Barcelona, España. Gedisa (1994)

(8) Freire, Paulo. A la sombra de este árbol. Madrid: Siglo XXI 1997

(9 ) Arandia L., Maite: La Formación de educadores y educadoras desde la mirada de Freire. ISSN 0213-8646. Revista Interuniversitaria de Formación del Profesorado., 18 (2), (2004),59-77.

(10) Pierce, Doris Ciencia de la ocupación y terapia ocupacional. Desenredando ocupación y actividad. AJOT, March- April 01 Vol 55 N² 2, pag 138-146

Traducción: TO Silvia Gómez

(11) Freire, Paulo. Pedagogía de la autonomía”. Madrid: Siglo XXI 1997. pág. 94.

(13) Rogers, C. y Mariam Kinget (1971) Psicoterapia y relaciones humanas. Tomo I Madrid- Barcelona Ed. Alfaguara. 